\title{
INTERPRETATION OF FATIGUE DAMAGE EVOLUTION IN RC SLABS BY MEANS OF INNOVATIVE 3D AE TOMOGRAPHY
}

\author{
T. SHIOTANI ${ }^{*}$ T. NISHIDA ${ }^{\dagger}$, H. ASAUE ${ }^{\dagger}$ AND Y. KOBAYASHI ${ }^{\dagger \dagger}$ \\ *Kyoto University \\ Nishikyo-Ku, Kyoto JAPAN \\ e-mail: shiotani.tomoki.2v@kyoto-u.ac.jp \\ ${ }^{\dagger}$ Kyoto University \\ Nishikyo-Ku, Kyoto JAPAN \\ e-mail: nishida.takahiro.6e@kyoto-u.ac.jp, \\ asaue.hisafumi.7a@kyoto-u.ac.jp, \\ ${ }^{\dagger}$ Nihon University \\ Chiyoda-Ku, Tokyo JAPAN \\ e-mail: kobayashi.yoshikazu@nihon-u.ac.jp
}

Key words: RC slab, Fatigue damage, Proactive maintenance, Q-value, AE tomography.

\begin{abstract}
The authors have been studying NDT based techniques that have potential to exhibit damage not only qualitatively but quantitatively. In the paper, two major aspects contributing to the damage have been introduced: a prospective damage index representing real internal condition; and damage visualization techniques with an acoustic approach, namely $\mathrm{AE}$ tomography. For a prospective damage index, Q-value, an attenuation feature of stress wave and can be treated as an irrespective parameter of frequency is demonstrated using several types of specimens changing the volume of artificial damage. And as for an innovative and the latest NDT technology, 3D AE tomography, which can visualize the internal 3D damage evolution, only from one side access situation, is introduced, followed by experimental verification using RC slabs subjected to cyclic mobile loads.
\end{abstract}

\section{INTRODUCTION}

Damage evolution of RC slabs in road bridges is regarded as the most difficult problem to implement rational maintenance. As the resultant investment from this fatigue damage shares a large portion in road budget, such measures have been conducted for this issue as from the basis of materials: improvement for the durability of concrete materials to resist the fatigue damage as well as to avoid cracking; and from the total cost for the life span: early damage detection with nondestructive testing (NDT) and corresponding reasonable repair works which enable reduction of life cycle cost.

Present study describes NDT based techniques that have a potential to exhibit damage not only qualitatively but quantitatively. Two major aspects contributing to the damage: prospective damage indices representing real internal condition and damage visualization techniques with an acoustic approach, namely Acoustic Emission tomography, are introduced. For a prospective damage index, Q-value, an attenuation feature of stress wave and can be treated as an irrespective parameter of 
frequency is demonstrated using several types of specimens changing the volume of artificial damage. And as for an innovative and the latest NDT technology, 3D AE tomography, which can visualize the internal 3D damage evolution, only from one side access situation, is introduced, followed by experimental verification using RC slabs subjected to cyclic mobile loads.

\section{IDEAL MAINTENANCE PROGRAM}

$\mathrm{RC}$ decks are damaging by repeated traffic load and the final form of the fatigue damage can be found by the evolutional cracks on the bottom side of concrete decks or pot hall on the asphalt pavement. As the current maintenance of those deteriorated $\mathrm{RC}$ decks have been conducted when the above surface damage becomes remarkable, repair works need a huge cost in comparison to of tiny damage generating inside of the deck in early phase of deterioration. At present it is an undoubted issue that proactive maintenance requires less cost that of corrective maintenance in a whole life of structure; however, there no decisive inspection approaches as to evaluate the tiny damage developed inside of the structure. In addition damage is also not well defined not only qualitatively but quantitatively in structural performance or integrity.

The authors have been thus studying the inspection methods being effective to the invisible damages as well as the damage indices, based on the elastic wave properties [1], [2]. Specifically in the following the damage index, namely Q-value is introduced, followed by the innovative visualization technique, $\mathrm{AE}$ tomography solving both of locations of $\mathrm{AE}$ sources as well as velocity distributions.

\section{DAMAGE DETECTION BASED ON Q-VALUE}

\subsection{Q-value}

So far the authors have studied the damage of the structures using such elastic wave methods as an $\mathrm{AE}$ technique and UT tomography. In the AE applications, we succeeded to relate the frequency features of
AE waveforms to damage [1], and in UT applications it has been clarified that those parameters as elastic wave velocity, an attenuation rate of amplitude, and frequency, could be indices of damage evaluation [3-6]. However, as for the velocity and the amplitude, it is known that the former are dependent on the frequency and the latter is readily decayed with propagation distance. The phenomenon of velocity depending on frequency is referred to as dispersion (character in which velocity changes with frequency), and the amplitude of detected waveforms is also dependent on the frequency. The amplitude of waveform is further influenced by the damping characteristic of the material and the propagation distance. Therefore, when associating these elastic wave parameters with deterioration, it is crucial to consider the frequency. As for the frequency component of the elastic waves, the high- frequency component decreases characteristically not only by the deterioration of a propagation medium but also by the increase of the propagation distance, suggesting that even for the case of the uniformly deteriorated materials, the frequency response would differ in the propagation distance. Accordingly it should be recognized that the frequency would not represent the only deterioration but obtained as a value resulted from several influencing factors. Q-value has a potential to be a damage index as it is an intrinsic attenuation rate being comparatively irrespective of the frequency and exhibiting quantitative values obtained from AE monitoring using plural numbers of sensors to some identified AE sources.

\subsection{Conceptual configuration}

AE waveforms are obtained as convolution of functions of source, propagation media, sensor and acquisition system in the time domain, and those frequency responses can be formulated by a simple multiple equation:

$$
X(f)=U(f) T(f) D(f) S(f)
$$

where $X(f), S(f), D(f), T(f)$, and $U(f)$ are Fourier transforms of detected $\mathrm{AE}$ waveforms, $\mathrm{AE}$ 
source, propagation media, sensor and acquisition system, respectively. $D(f)$ in Eq. 1 is a parameter which represent or quantify the damage. As $S(f)$ is not readily obtained in the AE technique, an approach to identify $D(f)$ irrespective to the $S(f)$ is crucial. In the $\mathrm{AE}$ application, multiple sensors are employed to locate the AE sources, and therefore the comparison of waveforms detected among different sensors for an AE source could suffice this requirement as in Eq. 2 assuming the frequency responses of all the sensors employed are compatible.

$$
\frac{X_{2}(f)}{X_{1}(f)}=\frac{T_{2}(f)}{T_{1}(f)} \cdot \frac{D_{2}(f)}{D_{1}(f)} \cong \frac{D_{2}(f)}{D_{1}(f)}
$$

On the other hand, as $D(f)$ is also dependent on the propagation attenuation, $D(f)$ can be defined as in Eq. 3 as well, where $f$ is a frequency $(\mathrm{Hz}), \mathrm{V}$ is a $\mathrm{P}$-wave velocity $(\mathrm{m} / \mathrm{s})$ and $\mathrm{Q}$ is a normalized value demonstrating attenuation rate.

$$
D(f)=\exp \left(-\frac{\pi f}{V Q} d\right)
$$

By combining Eq. 2 and Eq. 3, Eq. 4 is obtained.

$$
\frac{X_{i}(f)}{X_{1}(f)} \cong \frac{D_{i}(f)}{D_{1}(f)}=\exp \left(-\frac{\pi f}{V Q} \Delta d_{i}\right)
$$

Eq. 4 shows that a function of the ratio of frequency responses can be expressed by an exponential function composing from the difference of distance $\Delta d$ and frequency $f$. In this equation, using the waveform with a smaller propagation distance for the denominator of the ratio of a frequency response, $\Delta d$ always takes a positive value.

\subsection{Experimental procedures}

In this research, as for a preliminary phase to verify whether the concept of the ratio of frequency responses is applicable to the concrete, homogeneous standard mortar with formed polystyrene spheres simulating damage was used as test specimens. As shown in Figure 1 , the prism mortar specimens were prepared. All the sections were $150 \times 150 \mathrm{~mm}$ in section, but the height was different in each specimen: about 100, 200, 300, 400 and $500 \mathrm{~mm}$. In order to simulate the damage, spherical foamed styrene material (6 $\mathrm{mm}$ in diameter) was used as a false cavity, with four types of volumetric percentages as $0 \%, 1 \%, 5 \%$ and $10 \%$. Two types of elastic wave excitations were made by pencil lead break $(\sim 500 \mathrm{kHz})$ and electric pulser (PAC) through the wideband AE sensor (1045S, Fuji Ceramics Corp). Piezoelectric sensors of $60 \mathrm{kHz}$ resonant (R6, PAC) are employed for the receiver. The signals detected by the sensor were amplified by $40 \mathrm{~dB}$ at the amplifier, and processed and recorded by $\mathrm{AE}$ monitoring system (SAMOS, PAC) with $1 \mathrm{MHz}$ rate and $1 \mathrm{k}$ words samples. The elastic wave was excited at the center on the top surface of the specimen, and received by the sensor installed in the bottom surface.

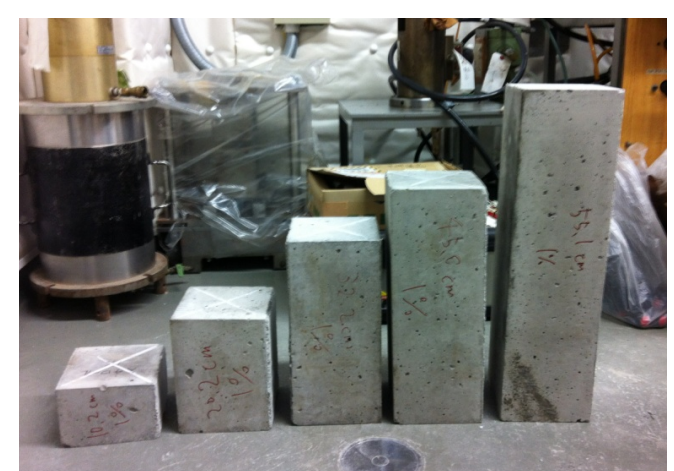

Figure 1: Mortar test specimens with simulated damages (Damage content 1\%).

\subsection{Results and discussions}

As a typical case, the result of damage content of 5\% is shown as in Figure 2. Figure 2 (a) shows the frequency distribution for the case of elastic wave excitation by electric pulser, and (b) shows that by pencil lead break. As can be found, the frequency spectra decrease with the increase of propagation distance, and the decrease rate is large as the frequency becomes high. It can be thus obvious that this result accords well to the theoretical formula as shown in Eq. 3 demonstrating the decay with the propagation distance. As for the difference of propagation distances, the attenuation trend 
as a function of frequency becomes remarkable when the propagation distance becomes longer even in the case of the same degree of damage ( $5 \%$ in this case), suggesting that it is not easy to evaluate degradation only by the frequency related parameters such as the centralfrequency and the peak-frequency which are well used to relate damage to elastic waves' parameters.

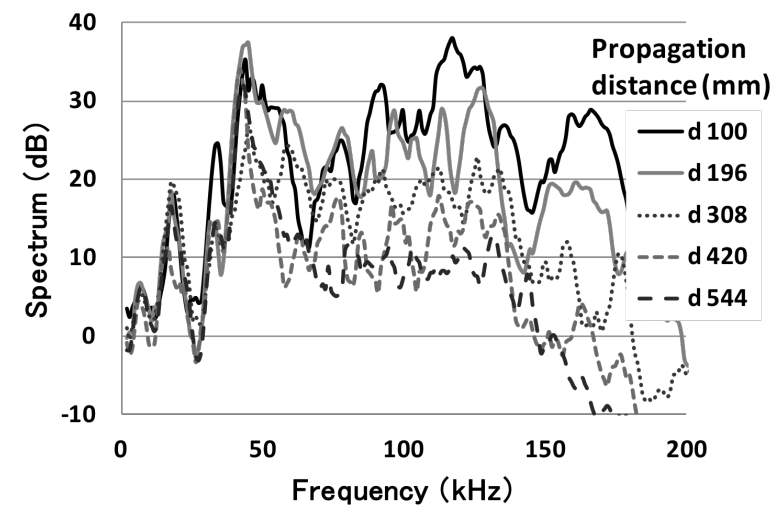

(a) Pulser

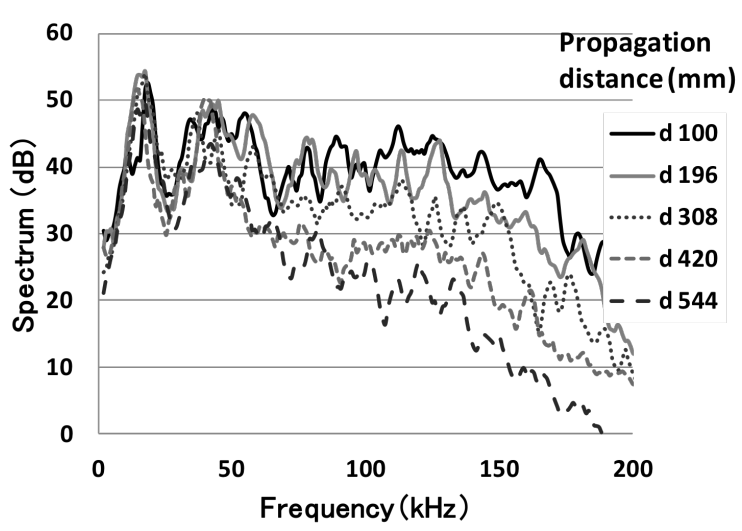

(b) Pencil lead break

Figure 2: Frequency distributions with different distances (damage content 5\%).

The ratio of frequency responses in terms of the difference of propagation distance from about $100 \mathrm{~mm}$ to $400 \mathrm{~mm}$ are obtained from the frequency response of $200-500 \mathrm{~mm}$ in propagation distances (in height of specimens) divided by one of a minimal $100 \mathrm{~mm}$ propagation distance for every damage degree. Specifically the ratio of frequency responses calculated as a function of the frequency can be found as in Figure 3. As in the way explained above, taking propagation distance $101 \mathrm{~mm}$ as a standard, the ratio of frequency responses with four distance differences $(96 \mathrm{~mm}, 208 \mathrm{~mm}$, $320 \mathrm{~mm}$, and $444 \mathrm{~mm}$ ) are obtained. Two straight lines in the figure show the linear approximation results over the frequency between 0 to $200 \mathrm{kHz}$ for the ratio of frequency responses of distance difference $96 \mathrm{~mm}$ and 444 $\mathrm{mm}$. In this experiment, as the same type of receiving sensor were used for all the measurement, $T_{2}(f) / T_{1}(f)$ in Eq. 2 can be regarded as 1 in all the frequency bands. Of course when two or more sensors are used for the measurement, one shall take each sensors' response into the consideration. Moreover, because of the heterogeneity of the mortar specimen, the ratio of frequency responses does not form a straight line as in a theoretical formula, and therefore, the regression line is used by carrying out linear approximation of the frequency response ratio.

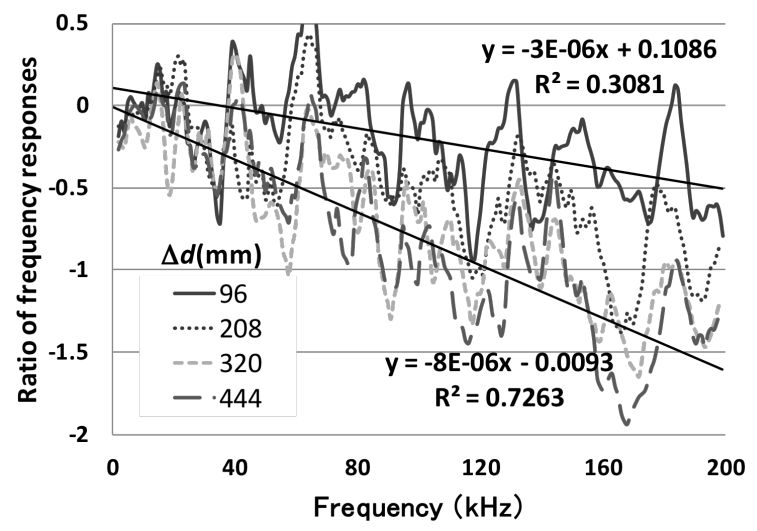

(a) Pulser

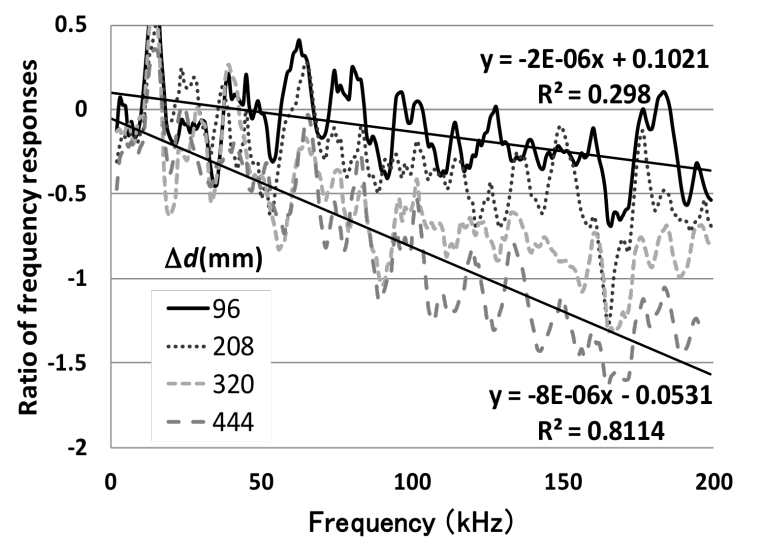

(b) Pencil lead break

Figure 3: Ratio of frequency responses with differences of propagations (damage content 5\%).

As found in Figure 3, the larger the difference of propagation distance takes, the larger the slopes of the ratio of frequency 
responses show. Here, it was verified experimentally that the theoretical formula, Eq. 4, well simulates the practical behavior. By using the slopes obtained from Figure 3, the ratio of frequency responses and the relation of a distance difference to each damage mixture rate are shown in Figure 4. As found, correlations were uniquely acquired in any damage mixture rate and in any excitations. The inclination obviously becomes large with the increase of damage volume. Then Q-value is determined by using Eq. 4, substituting P-wave velocity, $\mathrm{V}$, which was obtained by another measurement, and it is shown as in Figure 5.

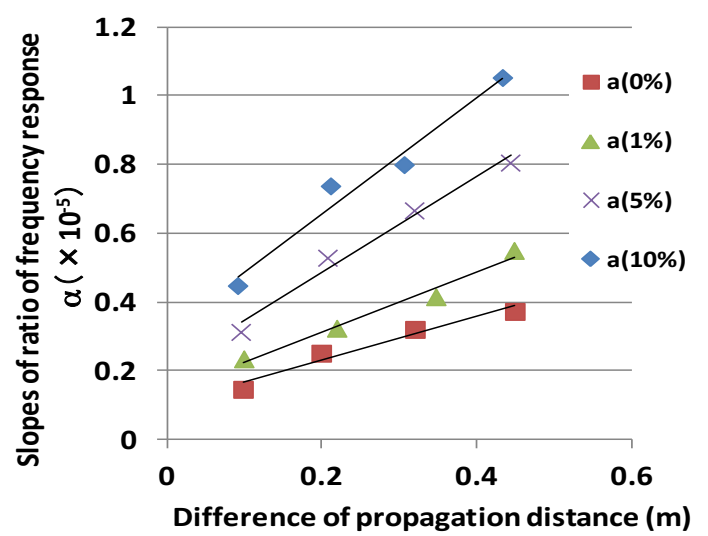

(a) Pulser

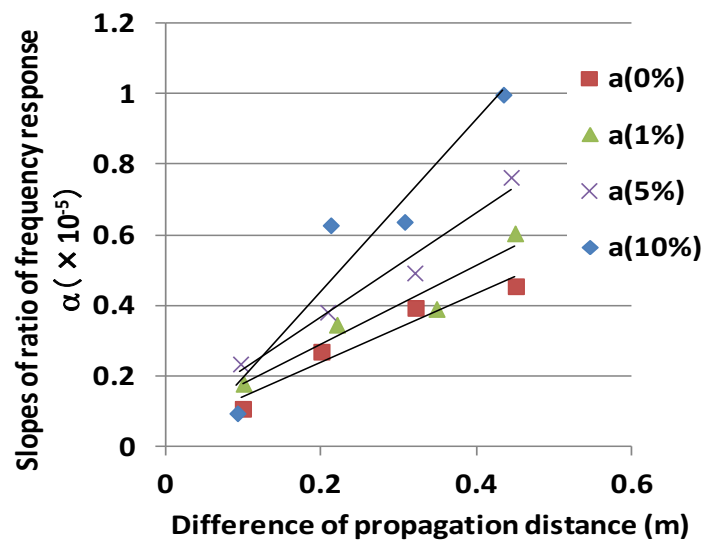

(b) Pencil lead break

Figure 4: Relation between slopes of the ratio of frequency responses and difference of propagation distances.

As mentioned already the velocity of a P-wave shows the dispersion depending on the frequency, e.g., the velocity becomes large to a certain frequency then decrease, and this tendency appears more remarkable when more damage was included [7]. In addition, in strictly
Q-value also depends on the frequency, showing the dispersion [8], the value shall be dealt with frequency. In fact, $Q$ values by two employed methods were different in the way of excitation as in Figure 5; however, it can be regarded as well that roughly the damage could be evaluated by Q-value, e.g., Q-value showing between 170-270 demonstrates intact condition of the material, 130 stands for $5 \%$, and about 100 for $10 \%$ damage. The dependency of $\mathrm{Q}$ value on the frequency and allowable range of Q-value will be clarified in the follow up paper.

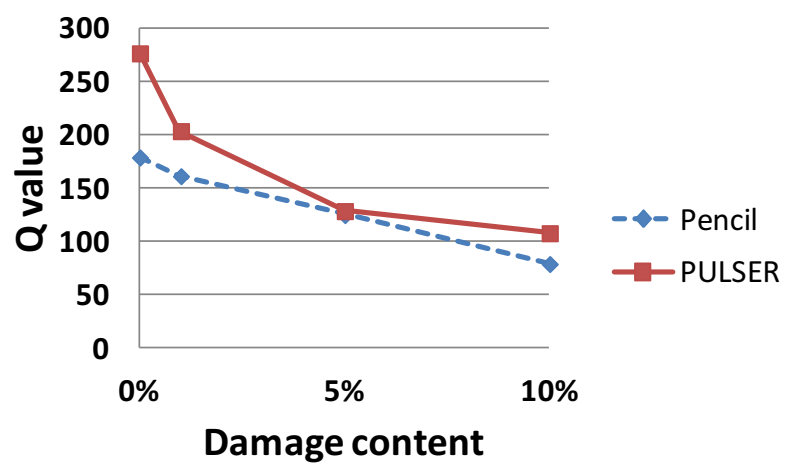

Figure 5: Q-values with damages for pulser and pencil lead break.

\section{FATIGUE DAMAGE EVOLUTION IN RC CONCRETE DECKS}

\subsection{Outline}

Among maintenance budget of road structures, remediation and replacement of RC bridge deck conquer the major part in Japan, and intensive studies to expand the service life of the deck are carried out. When implementing proper remediation program of the deck, evaluation of damage progress shall be examined in advance so that damage rating has been conducted by crack patterns by naked eyes. This way of investigation was well implemented for the correcting maintenance procedure where the repair shall be conducted when the remarkable deterioration was emerged on the surface; however, to reduce the repair budget, leading to decrease the amount of road investment, the deterioration/ damage shall be evaluated before reaching to the surface from the view point of life cycle cost [9]. Accordingly in this study, internal damage of 
$\mathrm{RC}$ deck due to fatigue failure is reproduced by wheel loading testing apparatus, and the damage progress was evaluated by $\mathrm{AE}$ measurement as well as 2D/3D AE tomography analysis.

\subsection{Wheel loading program}

To induce fatigue failure quantitatively, a RC deck specimen was subjected to repeated wheel loading with a steel wheel as shown in Figure 6. In the apparatus there is a steel wheel of 300 $\mathrm{mm}$ in diameter and $400 \mathrm{~mm}$ in width and can be applied the load up to $250 \mathrm{kN}$ in the case of dynamic and $534 \mathrm{kN}$ in the case of static load in the vertical direction. Contrary to the conventional wheel loading machine with a movable loading wheel [1], the foundation where the specimen is installed, is moved in the longitudinal direction within \pm 500 to \pm 1000 $\mathrm{mm}( \pm 500 \mathrm{~mm}$ in this test). The repetition rate can be set between $0.897-9.97 \mathrm{rpm} / \mathrm{min}$ (8.97 rpm in this test). The test specimen was $3000 \mathrm{x}$ $2000 \times 210 \mathrm{~mm}$ in size with steel reinforcement arranged as shown in Figure 7.

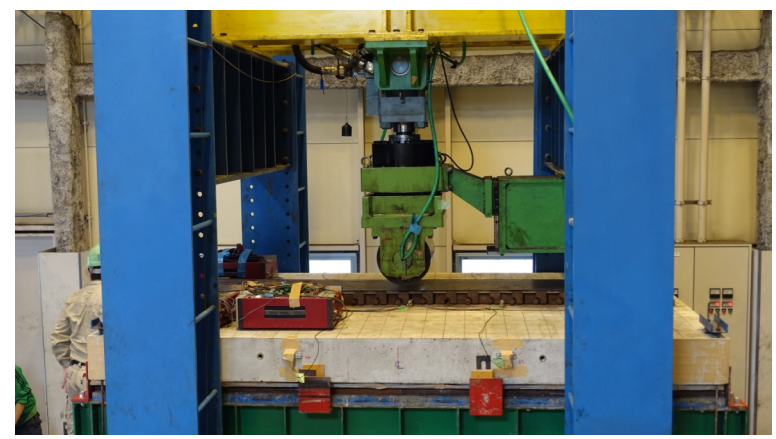

Figure 6: Wheel lading test machine.

Step-wise cyclic loading was performed as show in Figure 8. Firstly the load of $98 \mathrm{kN}$ was repeatedly applied for $100 \mathrm{k}$ times, followed by elastic wave excitations with $35 \mathrm{~mm}$ dia. steel hammer for elastic wave tomography. Subsequently AE monitoring with static load application to $98 \mathrm{kN}$ was conducted for the $\mathrm{AE}$ tomography. AE measurement was again conducted for the next loading step of $127.4 \mathrm{kN}$, and without $\mathrm{AE}$ measurement further load repetitions for $200 \mathrm{k}$ cycles were applied. Elastic wave excitations and $\mathrm{AE}$ monitoring were subsequently performed after this loading step with static load up to $127.4 \mathrm{kN}$. AE monitoring was then performed during the next loading of $156.8 \mathrm{kN}$. With this $156.8 \mathrm{kN}$ load, cyclic loading was conducted for $250 \mathrm{k}$ times. The same manner of elastic wave and AE measurements were repeated after this loading step, and the specimen was failed during the following loading step. Afterward cracks generated during the test were repaired with injecting epoxy-acrylate high-penetration resin. The agent was also expected to shield water infiltration from the surface into the specimen. After repair, elastic wave excitations were conducted for the tomography and then load application of the first applied i.e., the load application of $98 \mathrm{kN}$ was carried out with $\mathrm{AE}$ monitoring.

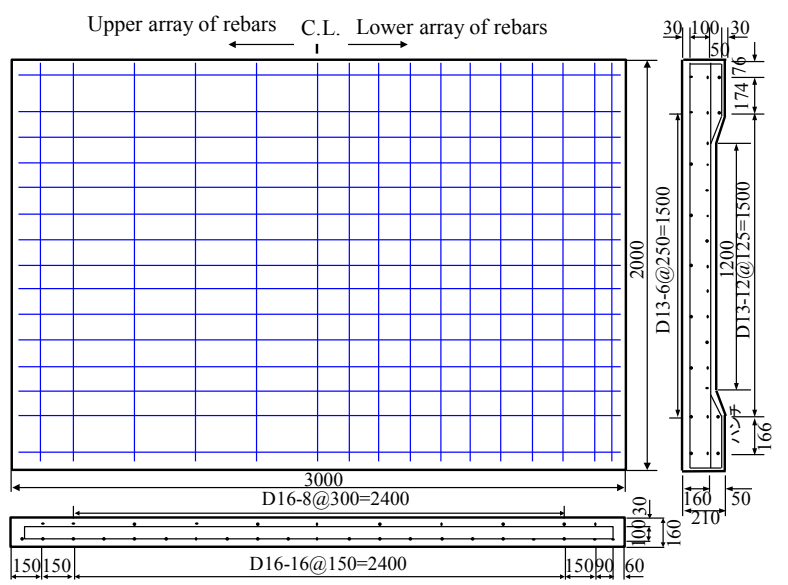

Figure 7: Configuration of RC deck specimen.

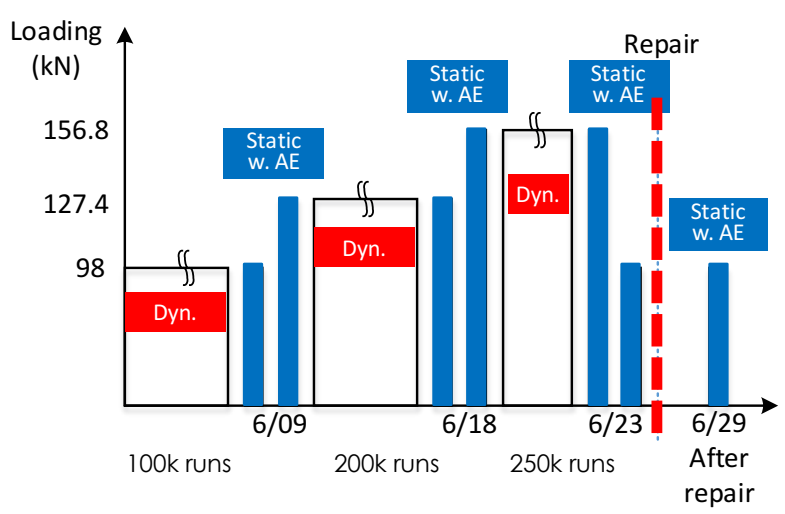

Figure 8: Load application program in wheel loading.

\subsection{Monitoring}

Damage evolution can be visualized when plotting accumulated AE sources through the whole failure process. In in-situ structures, however, as there are few cases installing $\mathrm{AE}$ 
sensors from default condition, cumulative damage interpretation with AE activity cannot be performed, and therefore tomographic approaches using through the thickness elastic waves have been employed. In conventional elastic wave tomography, the excitations shall be made at designated locations with accurate time record of excitation, leading to timeconsuming measurement and requiring a large numbers of sensors. For the bulky 3D structures of which no access allowed to the rear area, 3D visualization of damage, requiring sensors' placement on the rear side, cannot be implemented by the conventional tomography. As a solution for this difficult configuration, the authors' research group has proposed new approach namely 'Acoustic Emission tomography' (hereafter referred to as AET). In AET, calculation of both, source locations and velocity distributions are simultaneously implemented [2]. We've succeeded to develop it in three dimensions' problem recently [10].

In order to monitor AE activity as well as artificial excitations, $32 \mathrm{AE}$ sensors of $60 \mathrm{kHz}$ resonant were placed onto the four planes namely 10 on the top, 14 on the bottom and 2 each on the four sides. Artificial excitations were made by 35 dia. hammer at designated 12 points on the top and 14 points on the bottom surface. The wheel loads were applied in the longitudinal direction shown by the area surrounded by green lines as shown in Figure 9. In each phase-suspension of wheel loads, the specimen was subjected to static vertical loads at the central area shown by a blue rectangle. Both of AE signals and elastic wave signals due to excitations were amplified at the sensorintegrated pre-amplifier by $40 \mathrm{~dB}$ and acquired by AE monitoring system, 48-ch Express 8 of $\mathrm{PAC}$ with a 16 bit $\mathrm{A} / \mathrm{D}$ conversion rate and a 1 $\mathrm{MHz}$ sampling rate.

\subsection{AE tomography}

As shown above in AET, both of source locations and velocity distributions are simultaneously calculated with the following procedure. Hereafter, the source location algorithms are demonstrated, leaving detailed tomographic procedure to the other literature [11].

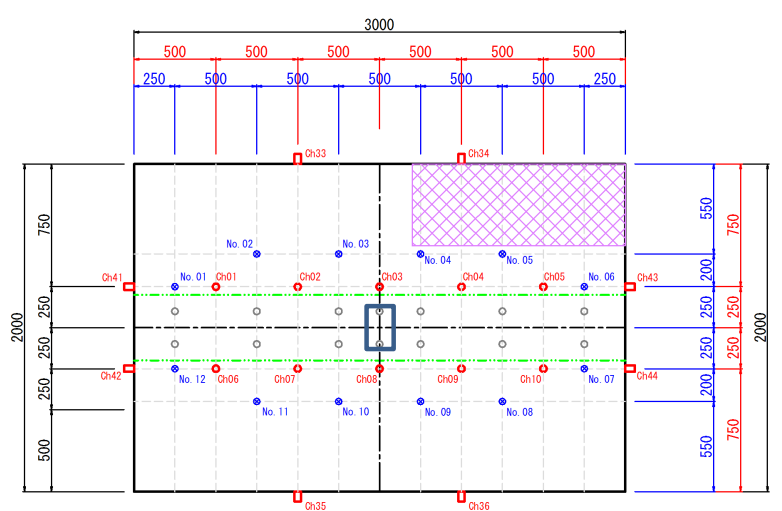

(a): Upper surface (unit: $\mathrm{mm}$ )

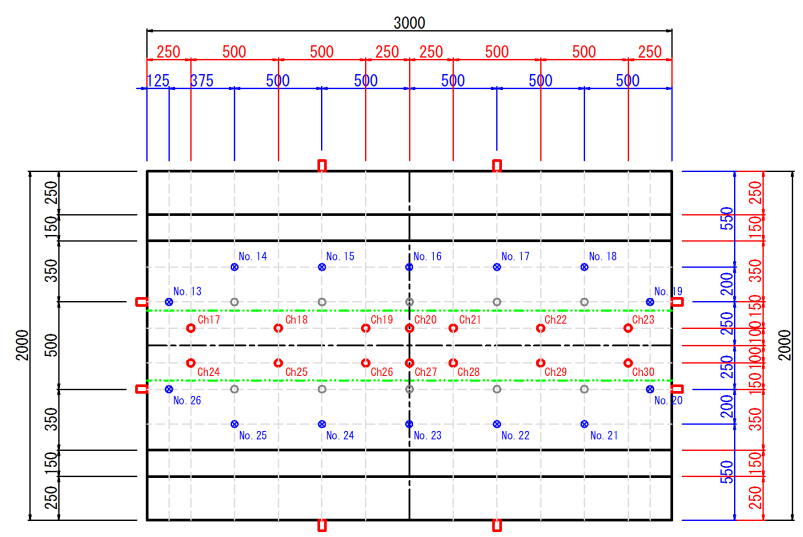

(b): Upper surface (unit: $\mathrm{mm}$ )

Figure 9: Arrangement of AE sensors.

The source location technique is based on ray-trace algorithm [12]. This algorithm is characterized by installation of relay points in each cell as illustrated in Figure 10. As the raypaths are formed by segments among nodal points in conventional ray-trace algorithm, its resolution depends on the mesh characteristics, implying that high accuracy source location requires fine mesh. This leads to increment of the number of degrees of freedom since slowness, which is a reciprocal of velocity, shall be defined in each cell, and consequently makes the identification procedure more complicated. In this ray-trace algorithm, the relay points between nodes are proposed and a ray-path is formed by segments among nodal, and relay points as shown in Figure 11. 


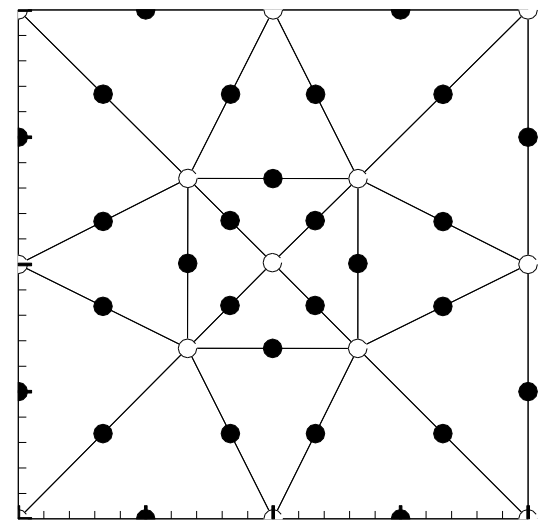

Figure 10: Conventional set of relay points.

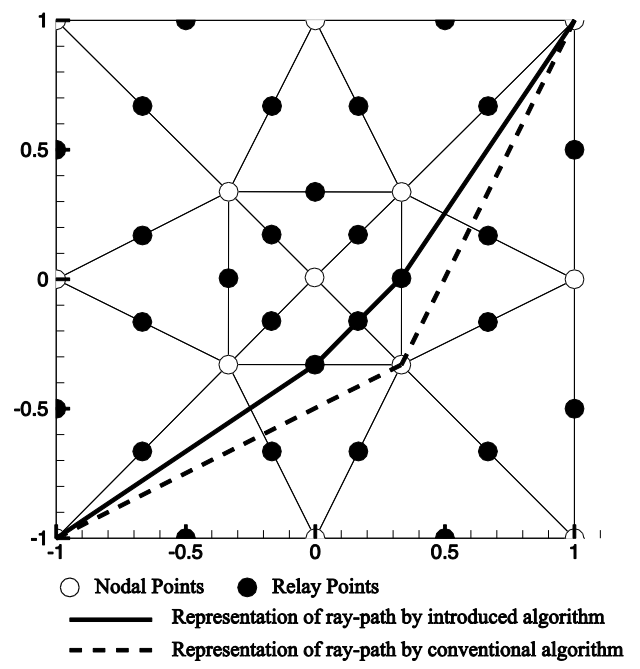

Figure 11: Revised ray path in consideration of proposed relay points.

The resolution of ray-trace is increased without increment of the degrees of freedom by this approach. Besides as role of the relay points is relaying the signals, the relay points shall be distributed uniformly on the surface. However, it is difficult due to the heterogeneous shape of cross section of concrete structures. To solve this problem, the relay points are installed by using iso-parametric mapping that is used in the ray-trace algorithm. Since each cell is mapped into isosceles right triangle, the relay points can be uniformly installed in the mapped cell as shown in Figure 12. This algorithm does not give exactly uniform distribution of relay points if the shape of the cell is skewed; however, the distribution is improved by avoiding use of strongly skewed cells. The source location is estimated by using this ray-trace algorithm.

The procedure of the estimation of source location is briefly described as in Figure 13. As for the first step to estimate the source location, the ray-trace is carried out for a receiver as illustrated in Figure 14.

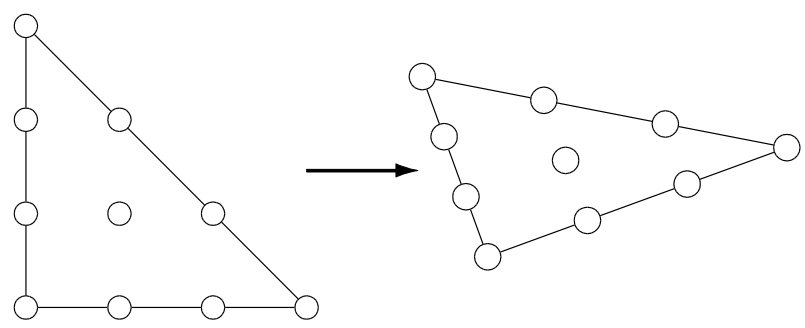

Figure 12: Mapping to the global coordinate of set relay points.

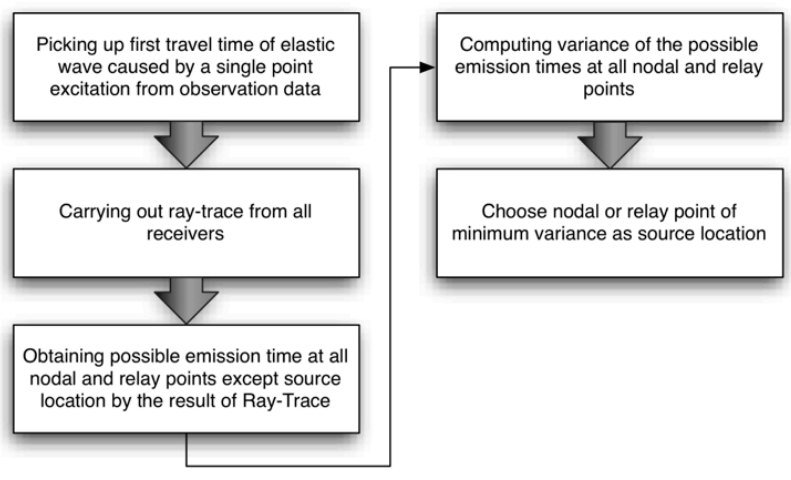

Figure 13: Procedure to estimate the source locations.

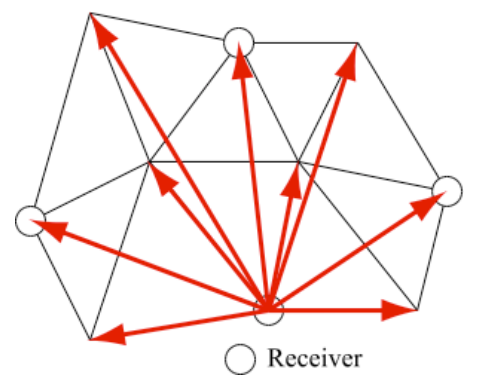

Figure 14: Ray-tracing from receiver to points.

This procedure calculates travel times $t_{i j}$ from a receiver $i$ to all nodal and relay points that are numbered as $j$. Since first travel time $T_{i}$ at receiver $i$ is observed, the possible emission time of the signal $E_{i j}$ is computed by Eq. 5 at a nodal or relay point $j$.

$E_{i j}=T_{i}-t_{i j}$

The step is applied for all receivers, and then variance of the $E_{i j}$ is computed as follow.

$\sigma_{j}=\frac{\sum_{i}\left(E_{i j}-m_{j}\right)^{2}}{N}$ 
in which

$m_{j}=\frac{\sum_{i} E_{i j}}{N}$

where $N$ is number of receivers. For the estimation of the source location, the variance $\sigma_{j}$ is evaluated. If the slowness distribution is exactly identical to real slowness distribution, $\sigma_{j}$ must be equal to zero at the source location and $m_{j}$ must be the emission time. Due to the discretization error of slowness distribution and insufficient resolution of ray-trace, $\sigma_{j}$ is not zero in general even at the source location while the identification procedure of seismic tomography. However, it is predicted that $\sigma_{j}$ gets minimum at the source location. Hence, in this procedure, the source location is determined as a nodal or relay point of minimum variance $\sigma_{j m i n}$. Additionally $m_{j}$ is used as possible emission time. It is noted that the accuracy of the estimation of source location relies on the density of nodal and relay points because the source location is assigned to a nodal or relay point in the proposed algorithm. Furthermore, by applying this technique to the iterative procedure of identification of wave velocity structure, the source locations are updated in every iterative step, improving the accuracy of the source location. This approach can be applied not only for AE signals but also signals that are generated by any excitation points.

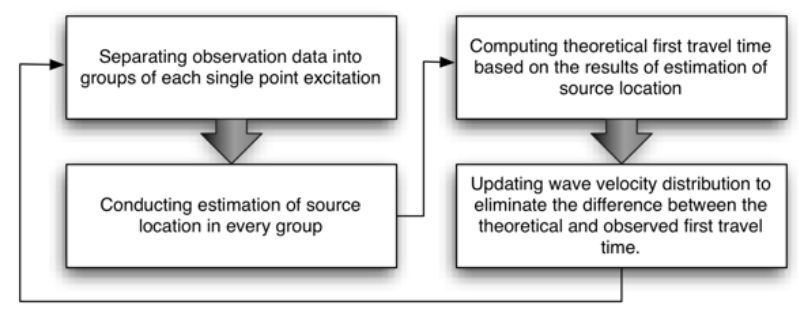

Figure 15: Procedure to estimate the source locations and velocity distributions.

Seismic tomography requires source location, emission time and travel time to the receiver. However, the signals having neither emission time nor source location can be used for seismic tomography. It is noteworthy that the source location and emission time can be estimated from travel times to the receivers under the wave velocity distribution determined by the method introduced in previous section. Based on these facts, a procedure of seismic tomography with estimation of source location is introduced. Figure 15 illustrates the procedure of seismic tomography with estimation of source location.

In the seismic tomography with estimation of source location, the first step is to estimate the source location and emission time. If the observed travel times can be separated into groups that are respectively associated with individual excitation points, the estimation of source location and emission time are carried out for each observed travel time group. The second step is applying the ray-trace to all estimated source locations. In this step, the raytrace is carried out for the all of estimated source locations, and the travel time among the estimated source locations to the other nodal or relay points are figured out. Finally by adding the computed travel time to the estimated emission time, the theoretical travel times at receivers are given by the following equation.

$T_{i}^{\prime}=m_{j}+t_{i j}$

In the third step, the slowness distribution is updated to eliminate the difference of the theoretical and observed travel time by identification technique.

In order to evolve this 2D AET to actual structures, 3D AET has been proposed [10]. Firstly the element in the 3D AE tomography is expressed in three dimensions, being different from the one of 2D AE tomography, where the most different point between 2D AET and 3D AET is the ray-trace technique in the algorism. In the developed ray-trace technique, the waves in 3D AET are expressed by Eqs. 9 and 10, which are expanded to three dimensions different from Eq. 11 used in 2D AET.

$$
\begin{aligned}
& a_{1} x+b_{1} y+c_{1} z+d_{1}=0 \\
& a_{2} x+b_{2} y+c_{2} z+d_{2}=0 \\
& a x+b y+c=0
\end{aligned}
$$


For the sake of this developed ray-trace technique, it can be possible to verify the $\mathrm{AE}$ source location and the deterioration of elastic wave velocity in 3 dimensions.

\subsection{Results and discussions}

The velocity distributions are referred to as 'tomogram,' and are exhibited for the case with artificial excitation and with $\mathrm{AE}$ sources by Figure 16a and Figure 16b, respectively. These are the $2 \mathrm{D}$ tomogram on the surface after the first load step, namely after 100k runs of $98 \mathrm{kN}$. As show in the both figures, remarkable damage could not be estimated by the velocity distributions. It is again noted that the tomogram was resulted from the $2 \mathrm{D} \mathrm{AE}$ tomography i.e., all the $\mathrm{AE}$ sources are projected on the top surface, and the velocity distributions are obtained based on the projected $\mathrm{AE}$ sources on the surface so that exact areas as well as source locations could not be discussed by these results.
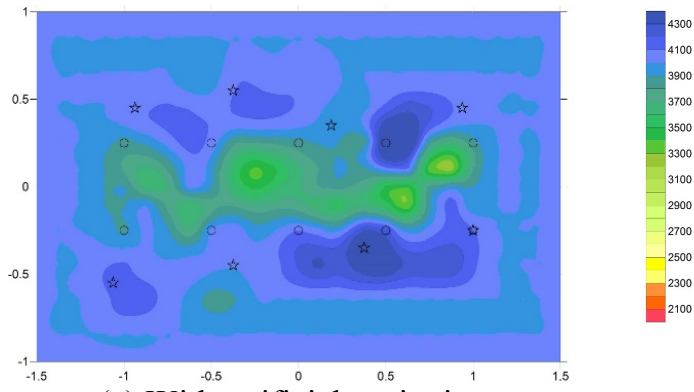

(a) With artificial excitations

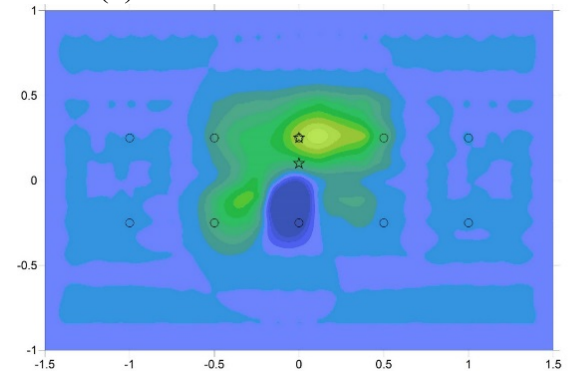

(b) With AE sources

Figure 16: Tomograms with two types of sources (2D) (figures are shown by the unit of meters/ second).

The tomogram using several AE sources during the following static load application of $127.4 \mathrm{kN}$ is demonstrated in Figure 17. At this step, obvious vertical damage areas are evaluated by the low velocity zones. For the subsequent loading steps the similar trends of low velocity zones are appeared in the tomograms. During AE monitoring after the $250 \mathrm{k}$ runs of $156.8 \mathrm{kN}$, intensive $\mathrm{AE}$ activity is observed around the central area of the specimen and resultant vertical cracks at -0.10 $\mathrm{m}$ and $-0.75 \mathrm{~m}$ in the longitudinal direction are emerged as show in Figure 18. Surprisingly these characteristics of damage form have already been estimated by the tomogram obtained during the load application of 127.4 $\mathrm{kN}$ (see Figure 17). It might be argued that there was no coincidence between the location of principal vertical crack generated in $-0.1 \mathrm{~m}$ in the longitudinal direction and the low velocity areas by the tomogram. This discrepancy seemed to be generated by two factors. One is that the tomogram was based on the projected AE sources to the top surface and exhibited one of the distributions within the specimen i.e., Figure 18 seemed to exhibit one of the internal and narrow damage area. The other is due to the form of principal vertical crack i.e., it seemed to be generated perpendicular to the surface; however, there possible to form a diagonal form in the thickness direction. Besides the central area, the low velocity area in Figure 17 was expanded to the left side in Figure 18, and therefore fatigue damage evolution due to cyclic loads was manifest by those tomograms.

The tomogram with artificial excitations after repair can be found as in Figure 19. Two expanded low velocity zones in Figure 18 were disappeared, and overall recovery of the velocity could be observed. The repair effect was thus verified with velocity distributions. After repair, a static small load of $98 \mathrm{kN}$ was applied to the specimen, and unexpectedly a large numbers of $\mathrm{AE}$ sources were acquired around the surroundings of the loading area as show in the stars of Figure 20. These AE activities appeared to be generated due to the failure of the thin repair agent on the surface. The resultant tomogram based on these $\mathrm{AE}$ sources accorded well with the AE activity. Low velocity zones are emerged around the loading plate (see Figure 9 a as for the location of the plate). From these findings it can be implied that the repair agent after the first load could not act as the protection for the water infiltration. 
In addition 3D AE tomography was conducted for the case after $200 \mathrm{k}$ runs as shown in Figure 21. In the figure, tomograms of top, middle and bottom planes are depicted with crack traces on the bottom. Although precise comparison of tomograms and crack developments could not be made as the tomograms are each representatives base on a series of prism elements, lower velocity areas coincide well with intensive cracked locations. As for the shape of damage e.g., cell-like crack patters can be well reproduced with low velocity distributions for the top and diagonal patterns of radiated cracks can be demonstrated with low velocity areas as well.

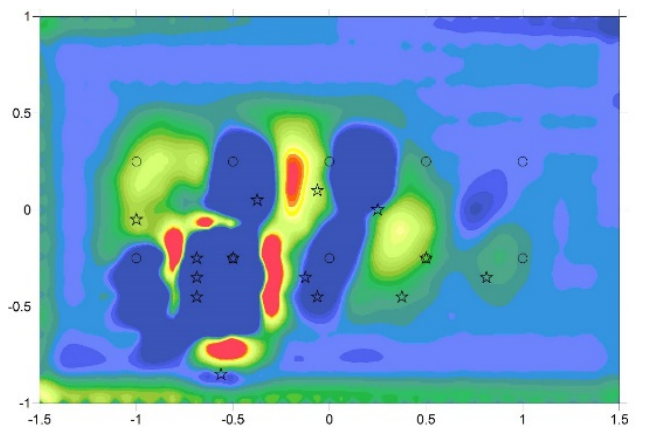

Figure 17: Tomogram with AE sources during load application of $127.4 \mathrm{kN}(2 \mathrm{D})$.
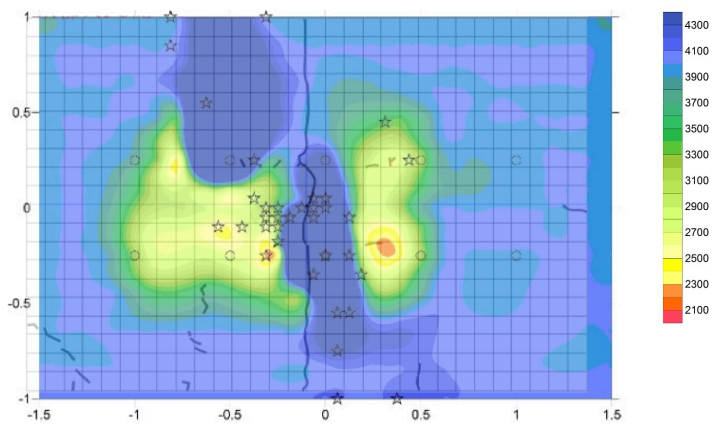

Figure 18: Tomogram with AE sources after 250k runs of $156.8 \mathrm{kN}(2 \mathrm{D})$.
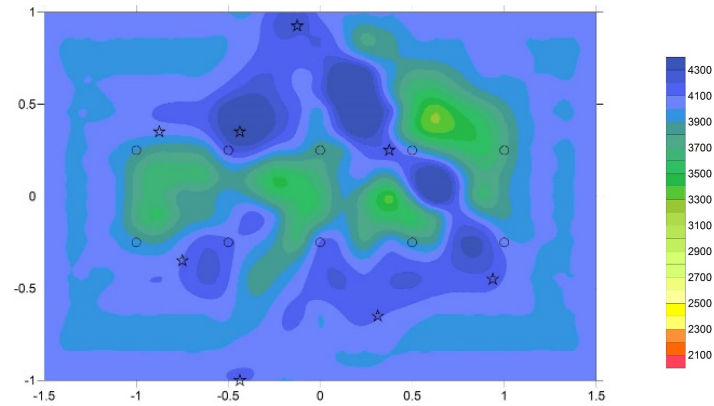

Figure 19: Tomogram with artificial excitations after repair $(2 \mathrm{D})$.

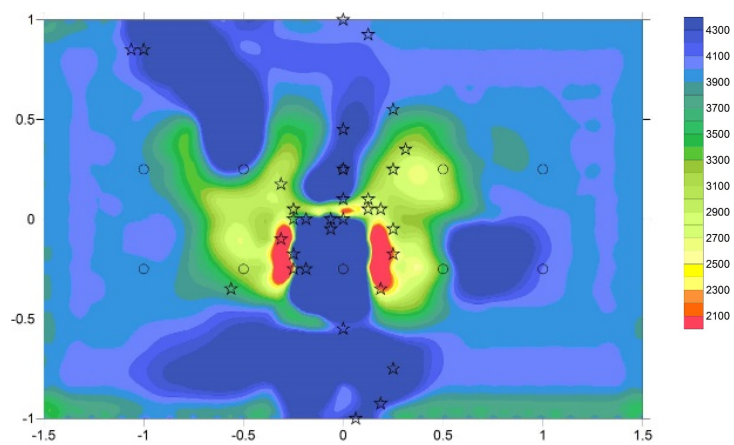

Figure 20: Tomogram with AE sources during the very first loading after repair (2D).

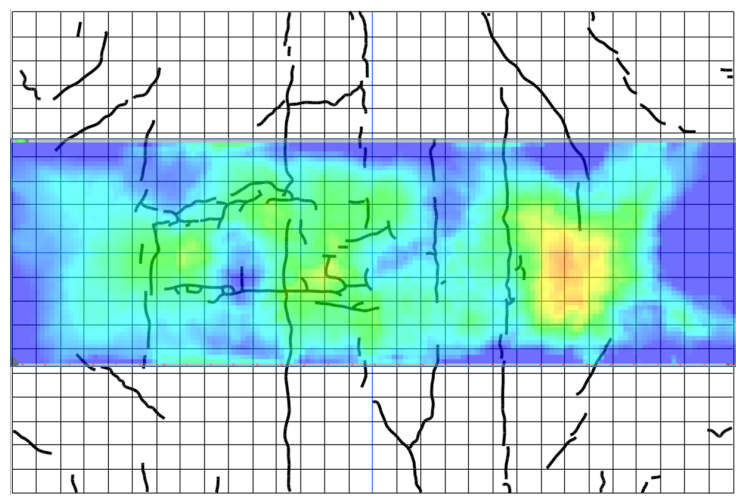

(a) Top surface with crack distribution

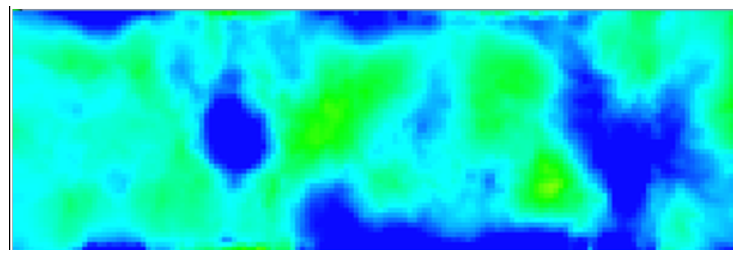

(b) Middle plane

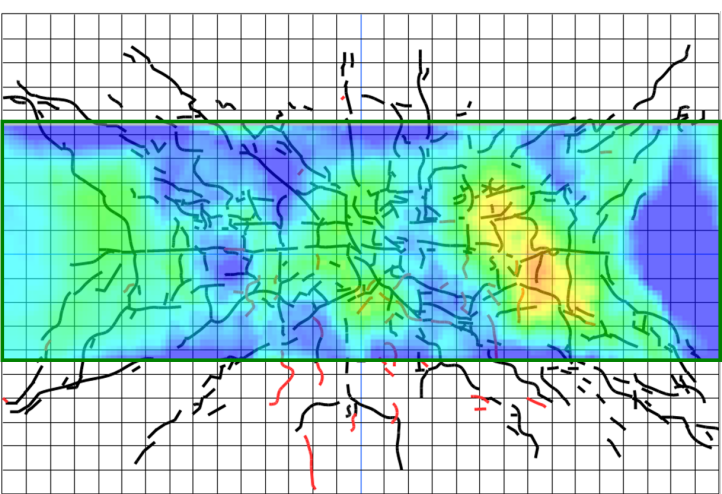

(c) Bottom surface with crack distribution

Figure 21: 3D AE tomography results at 3 different height planes after 200,000 times wheel loadings.

\section{CONCLUSIONS}

In order to study the quantitative evaluation method to assess the internal damage of the infrastructure with sufficient accuracy, $Q$-value 
analysis of $\mathrm{AE}$ waveforms was carried out. Through experimental works it can be concluded that $Q$-value could be a deterministic parameter to quantify the damage although allowable variation of $Q$-value and dependency on frequency shall be further studied.

Careful examination was conducted between developing cracks emerged on the surface and tomograms obtained from $\mathrm{AE}$ tomography during wheel loading tests of RC deck. As a result, tomograms obtained from 2D/ 3D AE tomography well demonstrated damages within the deck. In addition repair evaluation was performed with AE tomography, and it can be clarified that effectiveness of the repair works could be quantified with elastic wave velocities as well as their distribution. As the tomograms well evaluate internal condition both of before and after repair, this might be an essential tool to establish the life time scenario for concrete structures.

\section{ACKNOWLEDGEMENTS}

In this study, the experimental works of wheel loading test were conducted in College of Engineering of Nihon University under support of Cross- Ministerial Strategic Innovation Promotion Program (SIP) of Cabinet Office. Special thanks shall be sent to Prof. I. Iwaki and Associate Prof. Y. Koda with Nihon University as well as Prof. K. Maekawa and Associate Prof. Y. Tanaka with The University of Tokyo.

\section{REFERENCES}

[1] T. Shiotani, H. Ohtsu, S. Momoki, HK. Chai, H. Onishi and T. Kamada "Damage evaluation for concrete bridge deck by means of stress wave techniques," ASCE, J. Bridge Eng., Vol.17, No. 6, 2012, pp. 847-856.

[2] T. Shiotani, S. Osawa, S. Momoki and H. Ohtsu, "Visualization of damage in RC bridge deck for bullet trains with $\mathrm{AE}$ tomography," Springer, Advances in Acoustic Emission Technology, 2014, pp. 357-368.
[3] D.G. Aggelis, T. Shiotani, "Effect of inhomogeneity parameters on wave propagation in cementitious material," ACI Materials Journal, Vol. 105, 2, pp.187-193, 2008.

[4] D.G. Aggelis and T. Shiotani, "The influence of propagation path on elastic waves as measured by acoustic emission parameters," SAGE, Structural Health Monitoring, 11(3), pp. 359-366, 2011.

[5] W. I. Futterman, "Dispersive body wave," J. Geophys, Res., Vol. 67, No. 13, pp. 5279-5290, 1962.

[6] T. Shiotani, D.G. Aggelis, "Wave propagation in cementitious material containing artificial distributed damage," Materials and Structures 42, pp. 377-384, 2009.

[7] D.G. Aggelis et al., "Stress wave scattering: Friend or enemy of nondestructive testing of concrete?", Journal of Solid Mechanics and Materials Engineering, Vol. 2, No. 4, 2008.

[8] K. Yoshimura et al., "Experimental study on imaging hydraulic conductivity of rock masses based on elastic wave velocity dispersion," BUTSURI-TANSA, Vol. 62, No. 3, pp. 307-318, 2009.

[9] D. Frangopol, K. Lin and A. Estes, "LifeCycle Cost Design of Deteriorating Structures," ASCE, J. Struct. Eng., 07339445(1997)123:10(1390), 1997, pp. 13901401.

[10] T. Shiotani, S. Osawa, Y. Kobayashi and S. Momoki, "Application of 3D AE tomography for triaxial tests of rocky specimens," Proceedings of $31 \mathrm{st}$ conference of the European Working Group on Acoustic Emission (EWGAE), 2014, CD-ROM.

[11] Y. Kobayashi, T. Shiotani and H. Shiojiri, "Damage identification using seismic travel time tomography on the basis of evolutional wave velocity distribution model," Engineering Technics Press, Proceedings of Structural Faults and Repair 2006, 2006 (CD-ROM).

[12]F. Schubert, Basic principles of acoustic emission tomography, Journal of Acoustic Emission, 22, 2004, pp. 147-158. 